\title{
Identification of SCN7A as the key gene associated with tumor mutation burden in gastric cancer
}

Wenjie Li ${ }^{1,2+}$, Kezhi Zhou ${ }^{1,2+}$, Mengting Li ${ }^{1,2}$, Qian Hu ${ }^{1,2}$, Wanhui Wei ${ }^{1,2}$, Lan Liu ${ }^{1,2^{*}}$ and Qiu Zhao ${ }^{1,2^{*}}$

\begin{abstract}
Objective: Previous studies have shown that tumor mutation burden (TMB) in cancer is associated with prognosis. The purpose of this study is to identify TMB related genes in gastric cancer (GC) and to explore their prognostic value.

Methods: In our research, weighted gene coexpression network analysis (WGCNA) algorithm was used to cluster the most relevant TMB modules in the Cancer Genome Atlas (TCGA) database. Limma package was used to screen the differentially expressed genes, and the intersection was identified as hub genes. We used gene expression profiling interactive analysis (GEPIA) and survival algorithm to analyze the clinical characteristics and prognosis of hub genes in tumor and normal tissue samples of TCGA and Gene Expression Omnibus cohort respectively. We also used CIBERSORT algorithm to calculate the proportion of 22 tumor immune cells in the high and low expression subgroups of hub genes. In addition, we used gene set enrichment analysis (GSEA) to predict the biological function of hub genes. $P<0.05$ was considered statistically significant.
\end{abstract}

Results: In the TCGA cohort, TMB was significantly correlated with the clinical features of GC $(P<0.05)$. Through WGCNA and differential gene analysis, we identified SCN7A as the hub gene $(P<0.05,|\log 2 \mathrm{fc}|>1$, and $\mathrm{mm}>0.8)$. We found that the expression of SCN7A in tumor tissues was lower than that in normal tissues, and its expression level was also related to overall survival rate and tumor stage. GSEA analysis showed that SCN7A low expression group was enriched with "DNA replication", "base extension repair" and "proteasome" gene sets in GC. In addition, we found that there were significant differences in the infiltration degree of 7 kinds of immune cells between the two groups.

Conclusion: TMB can indicate the prognosis of gastric cancer. SCN7A is a hub gene associated with TMB, and its low expression is associated with better prognosis.

Keywords: Gastric cancer, TMB, SCN7A

\section{Introduction}

Gastric cancer is a malignant disease that occurred over 1 million and led to about 783,000 deaths worldwide in 2018, making it the fifth most common cancer and the third leading cause of tumor-related death [1]. With the

\footnotetext{
*Correspondence: Iliugi@whu.edu.cn; qiuzhao@whu.edu.cn

${ }^{\dagger}$ Wenjie Li and Kezhi Zhou contributed equally to this study

1 Department of Gastroenterology, Zhongnan Hospital of Wuhan

University, Wuhan, China

Full list of author information is available at the end of the article
}

improvement of diet and the screening and elimination of Helicobacter pylori, the incidence of gastric cancer has decreased. However, the GC incidence decreased among older adults and increased among younger individuals, especially in female adults [2]. The proportion of proximal gastric cancer, which usually leads to poor prognosis, has also increased. The current therapy for GC is primarily surgery resection combined with or without chemotherapy, which is limited. Hence, it is vital to find new strategies to improve prognosis. Although GC is a disease 
with high heterogeneity[3], a high incidence of somatic mutation occurred in GC patients [4]. Immunotherapy blocking immune checkpoints have achieved considerable progress in malignant diseases with increased somatic mutations, such as Hodgkin's lymphoma, melanoma and non-small cell lung cancer [5-7]. Thus, immunotherapy is a promising therapy for $\mathrm{GC}$.

Tumor mutation burden (TMB) is defined as the number of mutations, including replacement and insertion/ deletion, existing within a megabase of genomic territory [8]. It has been suggested by several studies that TMB is associated with the efficacy of immunotherapy, and patients with high TMB can benefit from immunotherapy and gain prolonged survival time in multiple cancer types [9-13]. However, immunotherapy does not suit every patient. At present, several clinical trials indicated that immunotherapy did not perform satisfactorily when treating GC patients [14, 15]. Therefore, finding novel biomarker for screening patients who are able to benefit from immunotherapy is of great importance.

The weighted gene coexpression network analysis (WGCNA) is a R package comprehensively collecting $\mathrm{R}$ functions for performing weighted correlation network analysis [16]. With the development of high-throughput sequencing technology, WGCNA is widely used to analyze data and microarray data to identity key genes in various diseases. For example, Nangraj, AS et al. applied the WGCNA to identity five hub genes between Barrett's esophagus and Esophageal adenocarcinoma [17].

In this study, TMB was calculated in 433 patients with GC in the TCGA database. Then, the WGCNA was utilized to identify hub gene associated with TMB and overall survival of GC patients. The Kyoto Encyclopedia of Genes and Genomes (KEGG) pathway enrichment analysis and Gene Ontology (GO) analysis were used to explore potential mechanisms. With the validation of external dataset GSE62254, we finally dig out the biomarker related to TMB in GC patients.

\section{Materials and methods Data preparation}

The human GC mRNA expression data and relevant clinical data were downloaded from the TCGA database (https://tcga-data.nci.nih. gov/tcga/). Somatic mutation data were also downloaded from the TCGA database. The GSE62254 GC mRNA expression data and corresponding clinical data were downloaded from (Gene Expression Omnibus) GEO (https://www.ncbi.nlm.nih. gov/geo/).

\section{Calculation of TMB in GC patients}

TMB is defined as the total number of mutations including somatic, coding, base replacement, and insert-deletion mutations per megabase in tumor tissue. 'maftools' R package was applied to calculate the TMB of each sample in TCGA-STAD, and the samples were divided into low and high TMB subgroups based on the median value. Moreover, we analyzed the difference between TMB high and low group among variant clinical characteristics.

\section{Co-expression network construction}

We calculated variances of 19,525 genes from "TCGASTAD." Ranked by decreasing standard deviation (SD), the top $25 \%$ of genes $(n=4881)$ were selected for further analysis. The "WGCNA" R package was used to construct a co-expression network of the above genes [18]. Further, the pickSoftThreshold function was used to estimate the soft-thresholding power $\beta$ for constructing modules. The parameter $\beta$ is a soft-threshold that can stress strong correlations between genes while penalizing weak correlations. According to the scale-free topology fit index and the mean connectivity (Additional file 1: Fig. S2 A-D), the value closest to 0.8 was chosen as the power to establish a scale-free network $\left(\beta=3\right.$, scale $\left.R^{2}=0.8\right)$. Next, to measure the network connectivity of genes, the adjacency was converted into a topological overlap matrix (TOM) [19]. Genes with similar expression patterns were classified into diverse modules with the smallest gene size of 30 based on the topological overlap matrix similarity. Eventually, the correlation between the module eigengenes and the clinic traits (overall survival, survival status, or TMB) was estimated to identify the correlative modules. Gene significance(GS) was defined as the absolute value of the correlation between genes and clinical traits. Quantitative measurement of the module relationship was determined as the connection strength between eigengenes in each module and gene expression profiles. The module significance (MS) was defined as the average GS for all the genes in the significant module. Moreover, module eigengene (ME) was the major component in the principal component analysis for each module, which could categorize all gene expression patterns into a single characteristic expression profile within a specific module. Based on the highest MS and the closest MEs correlation with $\mathrm{TMB}$, the key module was identified.

\section{Differentially expressed genes screening}

The 'limma' $R$ package was used to identify differentially expressed gene (DEGs) between normal and tumor samples [20]. Under the threshold of $p$-value $<0.05$ and $|\log 2 \mathrm{FC}|>1,304$ significant DEGs were selected for further analysis. 


\section{Functional enrichment analysis and hub gene identification}

To further investigate the function of DEGs in the key module, common genes in the key module and DEGs were exported. We used "clusterProfiler" R package to perform GO and KEGG enrichment analysis of the genes [21]. The enrichments of GO and KEGG analysis with $p<0.05$ were presented by using "ggplot2" $\mathrm{R}$ package. Moreover, the genes in the key module were ranked by decreasing module membership (MM) value and genes with $M M>0.8$ were identified as hub genes. hub genes were identified with high module membership (MM) which means genes were significantly related with the traits.

\section{Hub gene validation}

The survival curve was based on Gene expression profiling interactive analysis (GEPIA, http://gepia.cancerpku.cn/) with TCGA data. The overall survival analysis of GSE62254 was performed by "survival" $\mathrm{R}$ package. Expression of the hub gene between tumor and normal samples and among clinical traits were analyzed and visualized by GraphPad Prism software. The relationship between expression and clinical traits was evaluated by Chi-square analysis by using SPSS 25.0. The statistical significance was evaluated by two-tailed Student's t-tests. $p<0.05$ was considered statistical significance.

\section{Gene set enrichment analysis}

Three hundred and seventy-four samples were divided into high and low groups according to the expression of SCN7A. Gene set enrichment analysis was applied to enrich the KEGG pathways related to the two groups. | Normalized enrichment score $\mid>1.8$, p-value $<0.05$, and gene size $>30$ were used as the cutoff criteria.

\section{Evaluation of tumor-infiltrating immune cells}

The CIBERSORT algorithm was utilized to estimate the composition of tumor-infiltrating immune cells in normal tissues and GC tissues from the TCGA database [22]. The CIBERSORT algorithm containing 547 marker genes' expression signature matrix can be used to calculate 22 immune cells regarding LM22. LM22 is a txt defining 22 subtypes of immune cells referring to the annotated gene signature matrix, downloaded from the CIBERSORT website portal (Http:// cibersort.stanford.edu/).

\section{Results}

Tumor mutation burden spectrum and its correlation with clinical characteristics

We found that among 33 types of tumors, the TMB level of GC was relatively high (Additional file 2: Fig. S1A). The waterfall chart showed the top 30 mutated genes and related mutation types in TCGA-STAD (Fig. 1A). Missense mutations were the main type of mutation that occurred in GC patients (Fig. 1B). Single nucleotide polymorphisms occurred more than insertions or deletions (Fig. $1 \mathrm{C}, \mathrm{D}$ ), and $\mathrm{C}>\mathrm{T}$ was the common single-nucleotide variation (SNV) in GC. The frequencies of mutation in each sample were shown in Fig. $1 \mathrm{E}$ and $\mathrm{F}$. The barplot reveals the mutation of top 10 mutated genes (Fig. 1G).

To confirm the significance of TMB, we analyzed the relationship between TMB and clinical characteristics. The survival plot (Fig. 2A) showed that high-TMB was associated with better clinical outcome. TMB did not differ significantly among different gender, stage, grade, and Tumor Node Metastasis (TNM) (Fig. 2B, D-H). The level of TMB in patients $>60$ years old was remarkably higher than patients $\leq 60$ (Fig. $2 \mathrm{C}$ ).

\section{Co-expression network construction and key module identification}

We calculated the variances of 19,525 genes and chose 4881 genes with variances more than quartiles of variances for the subsequent analysis. R package "WGCNA" was applied to compute the 4881 genes into modules through the average linkage hierarchical clustering, and seven modules were finally presented with different colors (Fig. 3A). Among the modules, the maximum module contains 2387 genes (green). Moreover, the green module exhibits highest correlation with TMB $(\mathrm{r}=-0.33, p=7 \mathrm{e}-10)$ and significantly correlated with patients' survival status $(\mathrm{r}=0.12, p=0.03)$ (Fig. 3B). Figure $3 \mathrm{C}-\mathrm{E}$ showed the correlation between module membership and gene significance in the green module. The results indicated that the membership in the green module was significantly correlated with overall survival $(\mathrm{r}=0.37)$, patients' status $(\mathrm{r}=0.37)$ and TMB $(\mathrm{r}=0.74)$. Thus, genes in the green module were extracted for hub gene hunting.

\section{Hub gene identification and validation}

To further identify the hub gene, the threshold of $p$-value $<0.05$ and $|\log 2 \mathrm{FC}|>1$ were selected to identify DEGs. 153 upregulated genes and 151 downregulated genes were chosen for further analysis. 123 DEGs were also in the green module (Fig. 4A). To further evaluate the function of 123 DEGs, GO and KEGG pathway 
A Altered in $387(89.38 \%)$ of 433 samples.

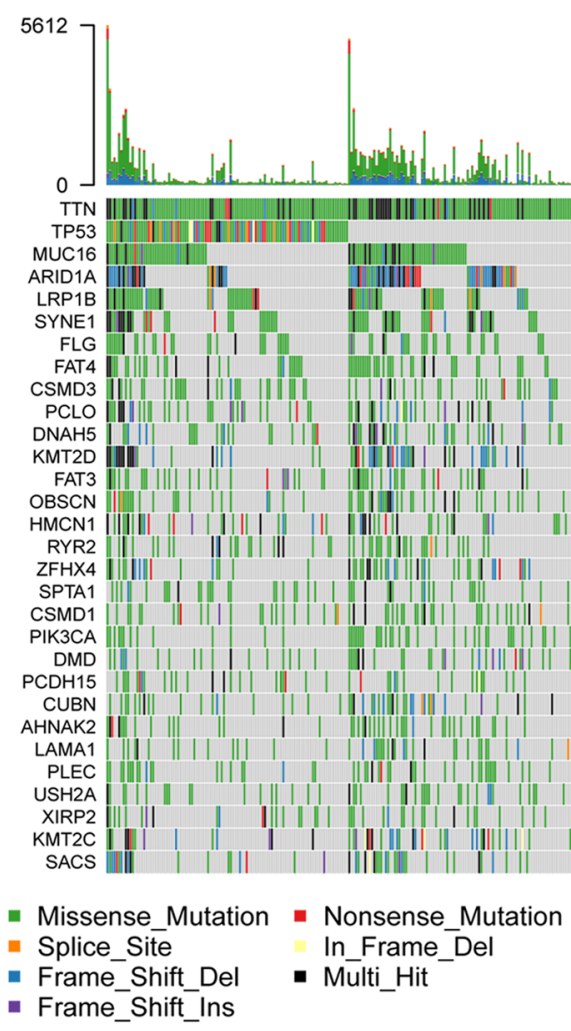

B

Variant Classification

C Variant Type

D SnV Class

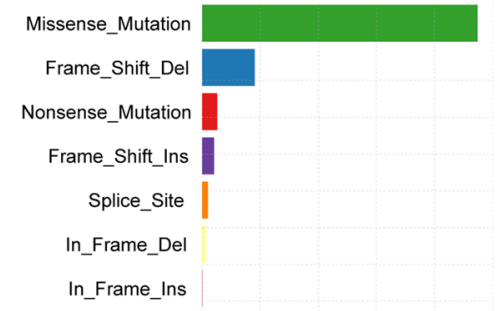

Nonstop_Mutation

Translation_Start_Site

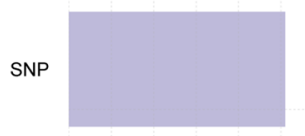

T>G 10536

T>A 5217

NS

T>C $\quad 26609$

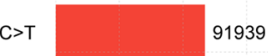

C>G 4761

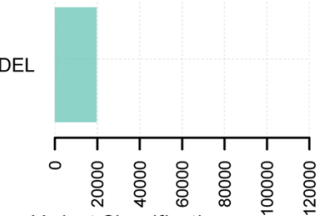

F Variant Classification

$C>A$

17847

E Variants per sample

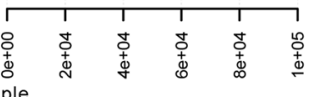

Median: 89
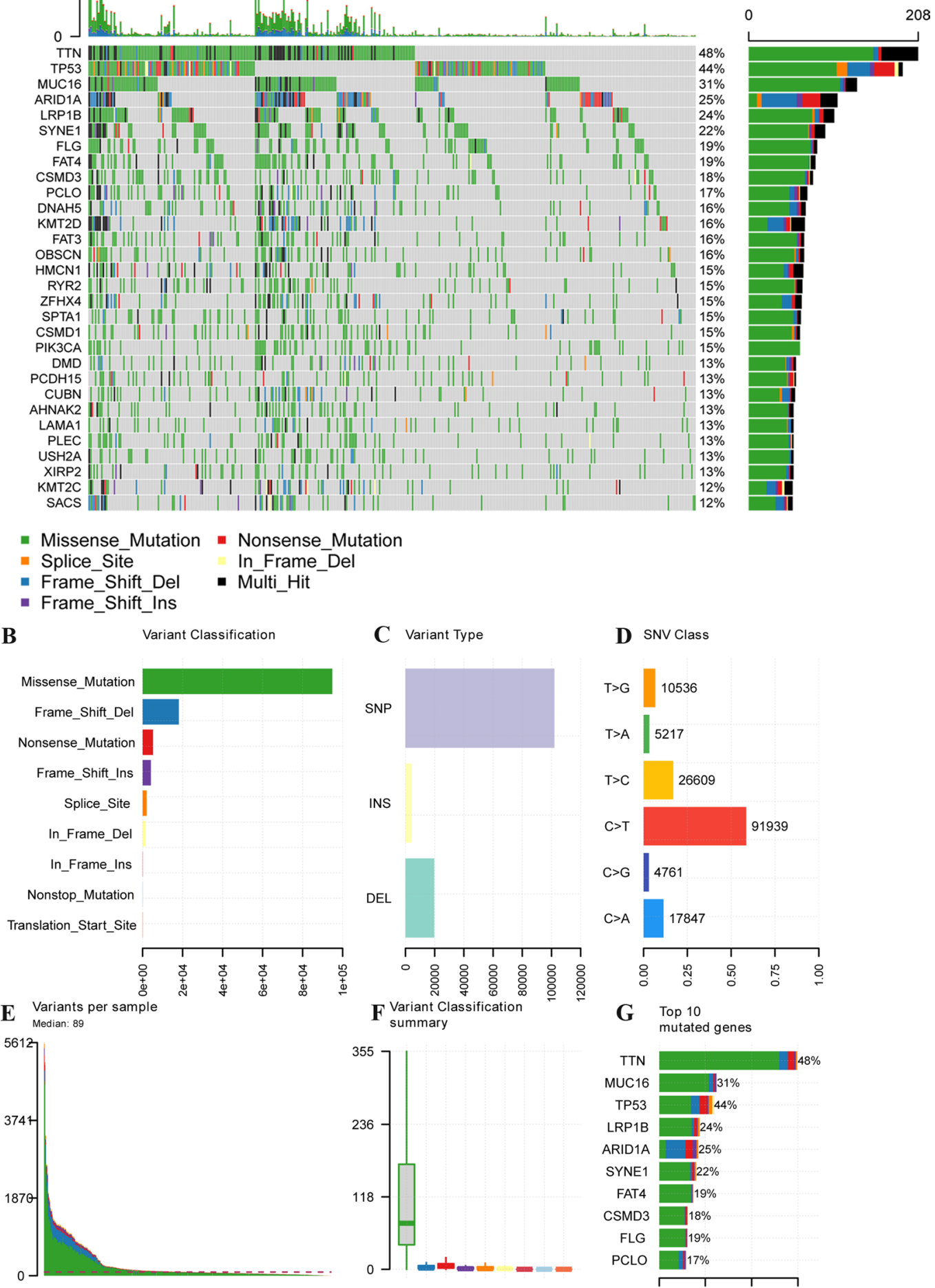

G Top 10

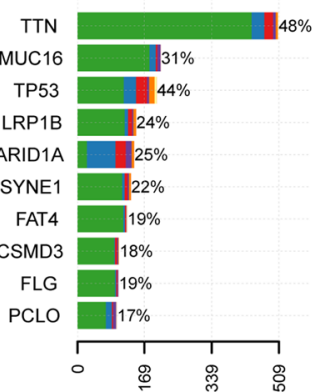

Fig. 1 TCGA GC mutation cohort. A Oncoplot depicts the frequently mutated genes in gastric cancer from TCGA cohort. The left panel shows mutation frequency, and genes are ordered by their mutation frequencies. The bottom panel presents different mutation types. B-G Landscape of TCGA GC cohort mutations 

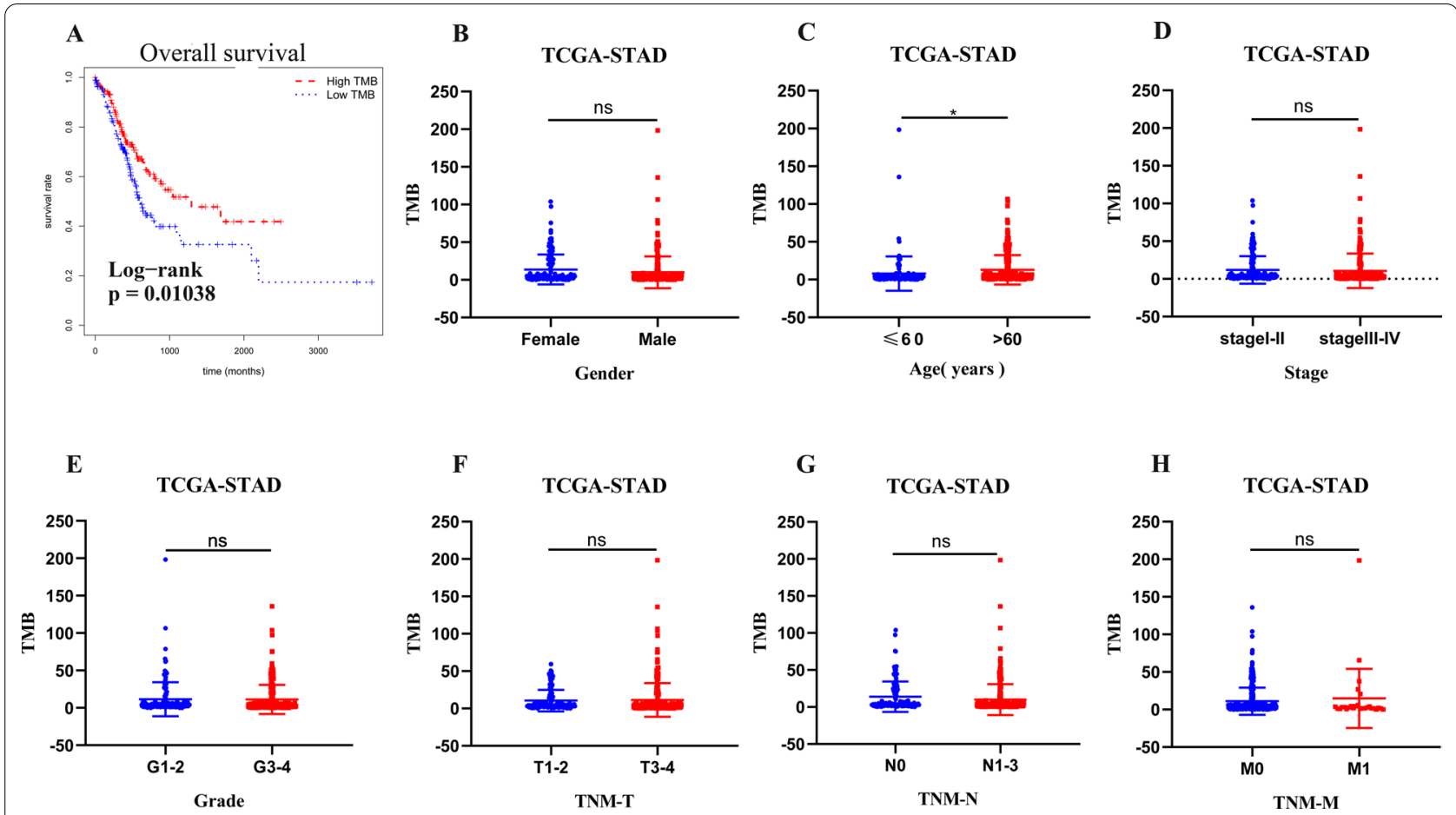

Fig. 2 Clinical significance of tumor mutation burden in gastric cancer patients. A Survival analysis to explore the overall survival of gastric cancer patients between the high and low tumor mutation burden groups. B-H Correlation between tumor mutation burden values and clinical characteristics in gastric cancer

analyses were performed. Protein digestion and absorption pathway was the most remarkably enriched KEGG pathway (Fig. 4B). "Extracellular matric organization" and "extracellular structure organization" were the most enriched in biological process (BP) while "collagen-containing extracellular matrix" was the most significantly enriched in cellular component (CC) and "receptor ligand activity" and "signalling receptor activator activity" in the molecular function (MF) (Fig. 4C).

Furthermore, to identify genes that were highly connected within a module, we set $M M>0.8$ as the threshold, and 5 genes were finally selected as the candidates (Fig. 4D). The survival plot by GEPIA revealed low expression level of SCN7A was associated with overall survival rate (Fig. 4E, $p=0.043$ ) and further validated by external dataset GSE62254 (Fig. 4F, $p=0.026$ ). Thus, SCN7A was identified as the hub gene. Furthermore, we explored the relationship between SCN7A and clinical characteristics in GSE62254. The expression level of SCN7A in tumor tissues was lower than normal tissues (Fig. 5A). We also found that the expression of SCN7A was lower in stage I-II compared with stage III-IV (Fig. 5B) while it is higher in patients with T3-4 (Fig. 5C). In addition, the samples in GSE62254 were divided into two groups (high and low) by SCN7A average expression level. The relationship between clinical traits and the expression level of SCN7A was analyzed. As shown in Table 1, the expression level of SCN7A was related with gender, age, TNM-T, TNM-N and stage (with $p<0.01$ ). To investigate the potential biological function of SCN7A in GC samples, we performed GSEA and with the threshold of $|\mathrm{NES}|>1.8$ and $\mathrm{NP}<0.05$, the gene set of "DNA REPLICATION", "BASE EXTENSION REPAIR" and "PROTEASOME" was enriched in SCN7A lowly expressed samples (Fig. 5D-F). In summary, our results indicated that SCN7A was associated with clinical characteristics and TMB to a large extent.

\section{Relationship between hub gene and tumor infiltrating immune cells}

Study suggested that TMB was related to the efficacy of immunotherapy in most cancer types. Hence, we calculated the fraction of tumor infiltrating immune cells in GC through CIBERSORT algorithm. The samples in TCGA were divided into two groups (high and low) by average expression of SCN7A. Figure 6A indicated that the fractions of several immune cells were different between SCN7A low (blue) and high (red) samples. $B$ cells naïve, $T$ cells $C D 4$ memory resting, $T$ cells $C D 4$ memory activated, $\mathrm{T}$ cells follicular helper, Macrophages M0, Mast cells resting, and Mast cells activated differed significantly between SCN7A low and high groups. To 


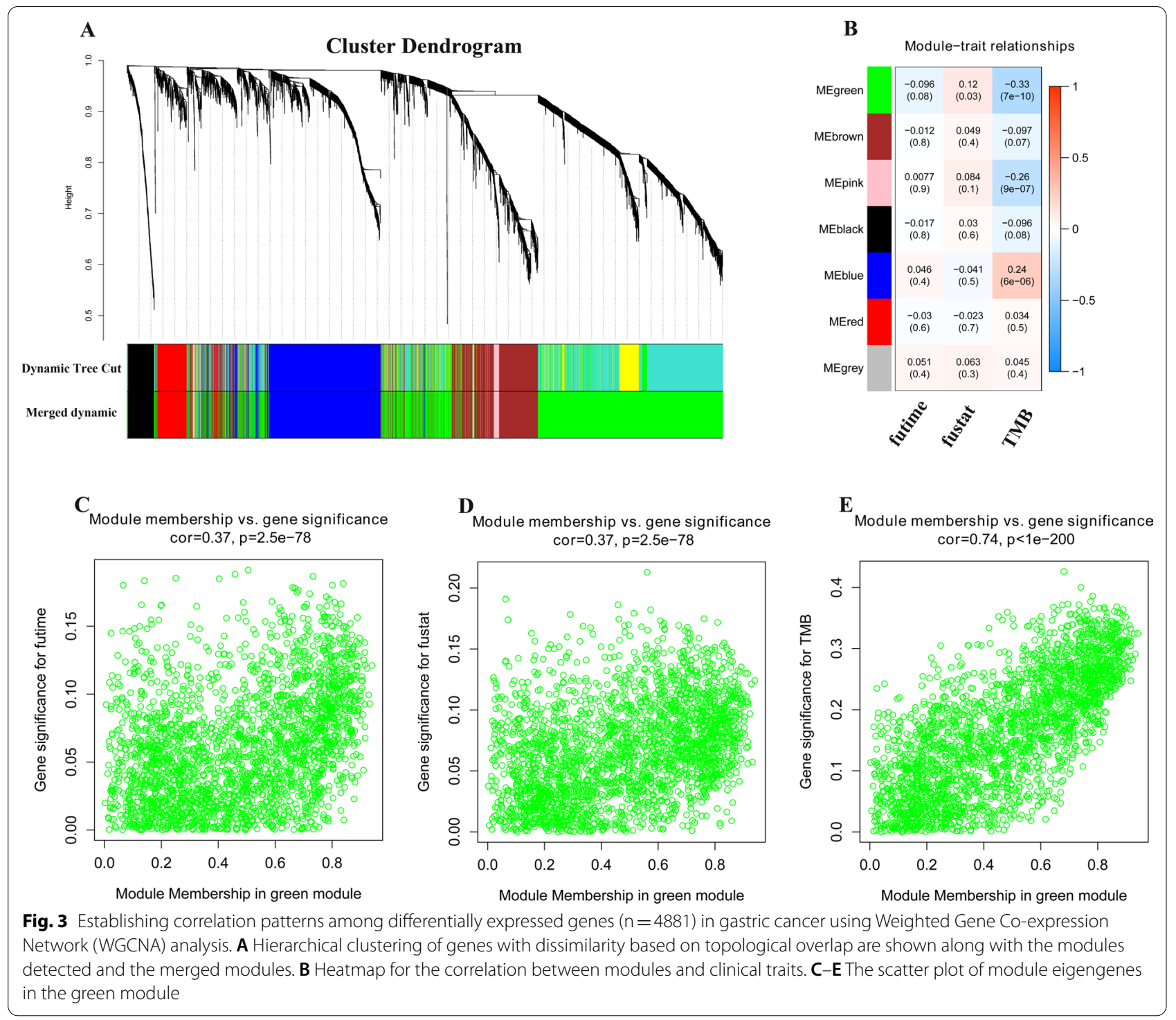

further analyze the effects of SCN7A in tumor-infiltrating immune cells, we calculated the correlation between SCN7A expression levels and TIICs. The expression level of SCN7A was positively correlated with the fraction of $\mathrm{T}$ cells CD4 memory resting (Fig. 6B), monocytes (Fig. 6E), and mast cells resting (Fig. 6G) while negatively correlated with fractions of $\mathrm{T}$ cells $\mathrm{CD} 4$ memory activated (Fig. 6C), T cells follicular helper (Fig. 6D), and macrophages M0 (Fig. 6F).

\section{Discussion}

Gastric cancer is a disease leading to numerous deaths worldwide. Finding a biomarker which is enable to predict immune response in gastric cancer has great guiding significance for the treatment of tumors, with the development of immunotherapy and wide application in numerous neoplastic diseases. Currently, the prevailing view is that TMB can reflect the therapeutic effect of chemotherapy drugs on cancer to some extent, especially immune checkpoint inhibitors. For example, in patients with non-small cell lung cancer with high TMB $(\geq 10$ mut/MB), anti-PD-1/PD-L1 immunotherapy results in excellent outcomes regardless of PD-L1 expression levels In this study, we found that TMB of gastric cancer was the fourth highest among all kinds of cancer by bioinformatic analysis based on TCGA database, implying that GC could be considered as an immune-responsive tumor. Then we used WGCNA to cluster 4881 genes and got a module highly correlated with TMB $(\mathrm{r}=-0.33$, $p=7 \mathrm{e}-10)$. In this module, we were interested in the 

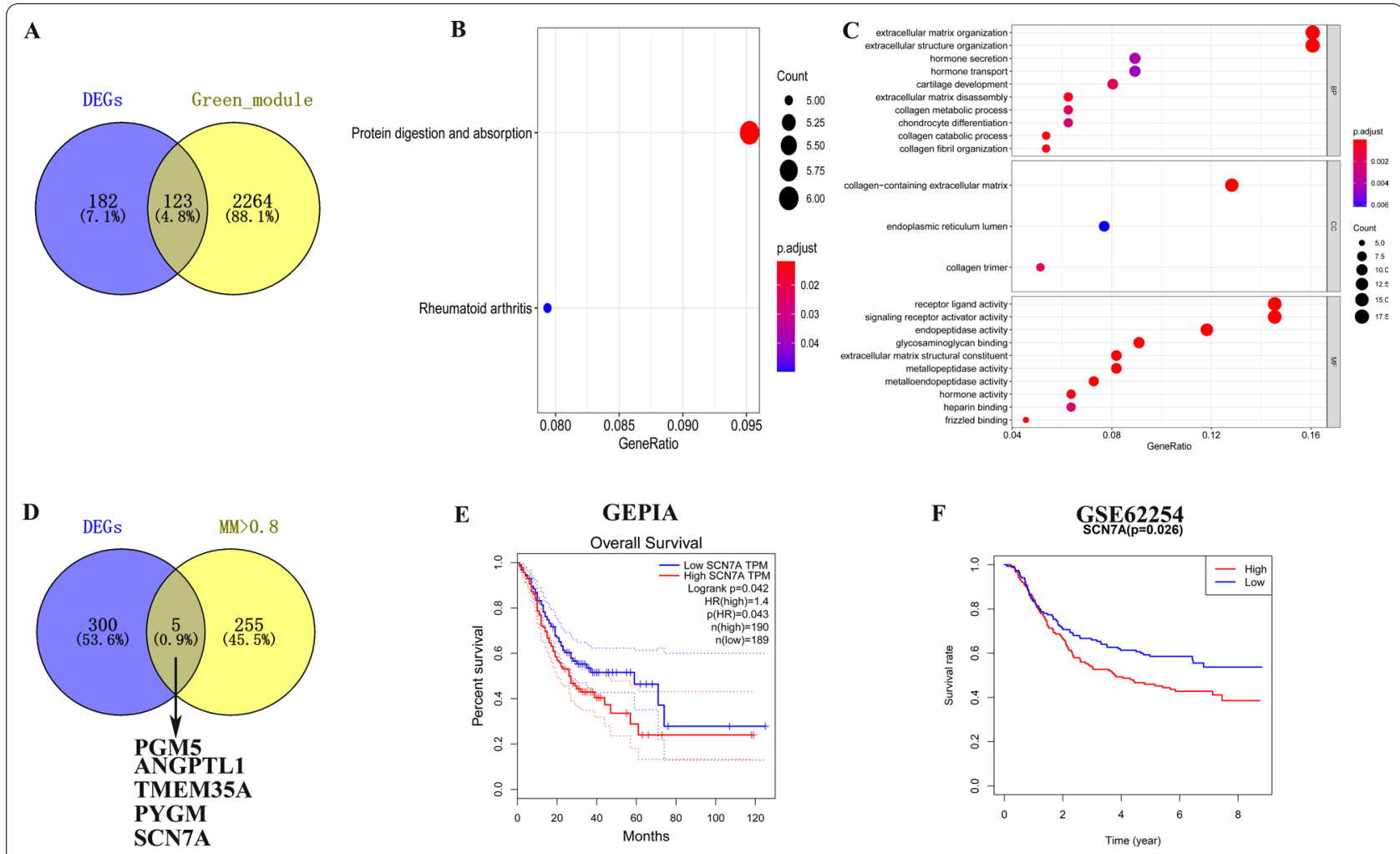

Fig. 4 A Overlap of green module hub genes and DEGs genes. B KEGG pathway enrichment analysis. C GO pathway enrichment analysis. D Overlap of DEGs genes and green module genes (MM > 0.8). E, F The overall survival of patients with high and low expression levels of SCN7A by GEPIA and in GSE62254

selected hub gene SCN7A. According to the results of KEGG pathway analysis, we inferred that SCN7A could affect the digestion and absorption function of gastric epithelial cells. Meanwhile, based on the existing clinical data of $477 \mathrm{GC}$ patients from TCGA database and the microarray of GEO database, the mRNA level of SCN7A was negatively correlated with its clinical prognosis, and the later the clinical stage of the patients, the higher its expression was. Moreover, Tumor immune cell infiltration results showed that low-expression group of SCN7A had more infiltration in $\mathrm{T}$ cells $\mathrm{CD} 4$ memory resting and macrophages $\mathrm{M} 0$, while less infiltration in $\mathrm{T}$ cells CD4 memory activated and monocytes, indicating that such immune cells and pathways play a dominant role in the tumor microenvironment and promote immune responses.

Sodium Voltage-Gated Channel Alpha Subunit 7 (SCN7A) encodes a protein that is a specific sodium channel [23]. The SCN alpha family encodes proteins which are alpha subunits of sodium channel, called $\mathrm{Na}_{\mathrm{v}} 1$ [24]. Voltage-gated sodium channels are essential for initiating and propagating action potentials after depolarization of the cell surface membrane in most neurons [25]. The SCN7A protein is called $\mathrm{Na}_{\mathrm{x}}$ for not being voltage gated. But SCN7A appears to be the most basal part of the SCN alpha family [26]. By locating on the plasma membrane, ion channels can sense and respond to changes in the extracellular environment, thereby playing a key role in cell signal transduction and cancer progression [27]. Studies have identified $\mathrm{Na}_{\mathrm{x}}(\mathrm{SCN7A})$ as a specific sodium sensor mainly distributed in skin and other epithelial cells. Its upregulation mostly occurs in scars and skin inflammation and can activate the major sodium channel $\mathrm{ENaC}$ as well as the downstream inflammatory signal prostaglandin E2, while also regulating other pathways such as PAR-2 to promote the release of inflammatory factors IL-1 $\beta$ and IL-8 [28]. Among them, IL-8 presents an inhibitory effect on adaptive immunity, affecting antigen presentation and the antitumor activity of effector T cells. The proportion of IL-8-producing myeloid cells and lymphocytes was higher in the resistant patients compared to those responding to immunotherapy, suggesting that high IL-8 is likely to result in suppression of antitumor immunity $[29,30]$. In our study, we found that low expression of SCN7A was related to an increase in TMB. Tumors with high TMB imply the exposure of more neoantigens and thus are more prone to trigger $\mathrm{T}$ cell dependent immune responses to inhibit 


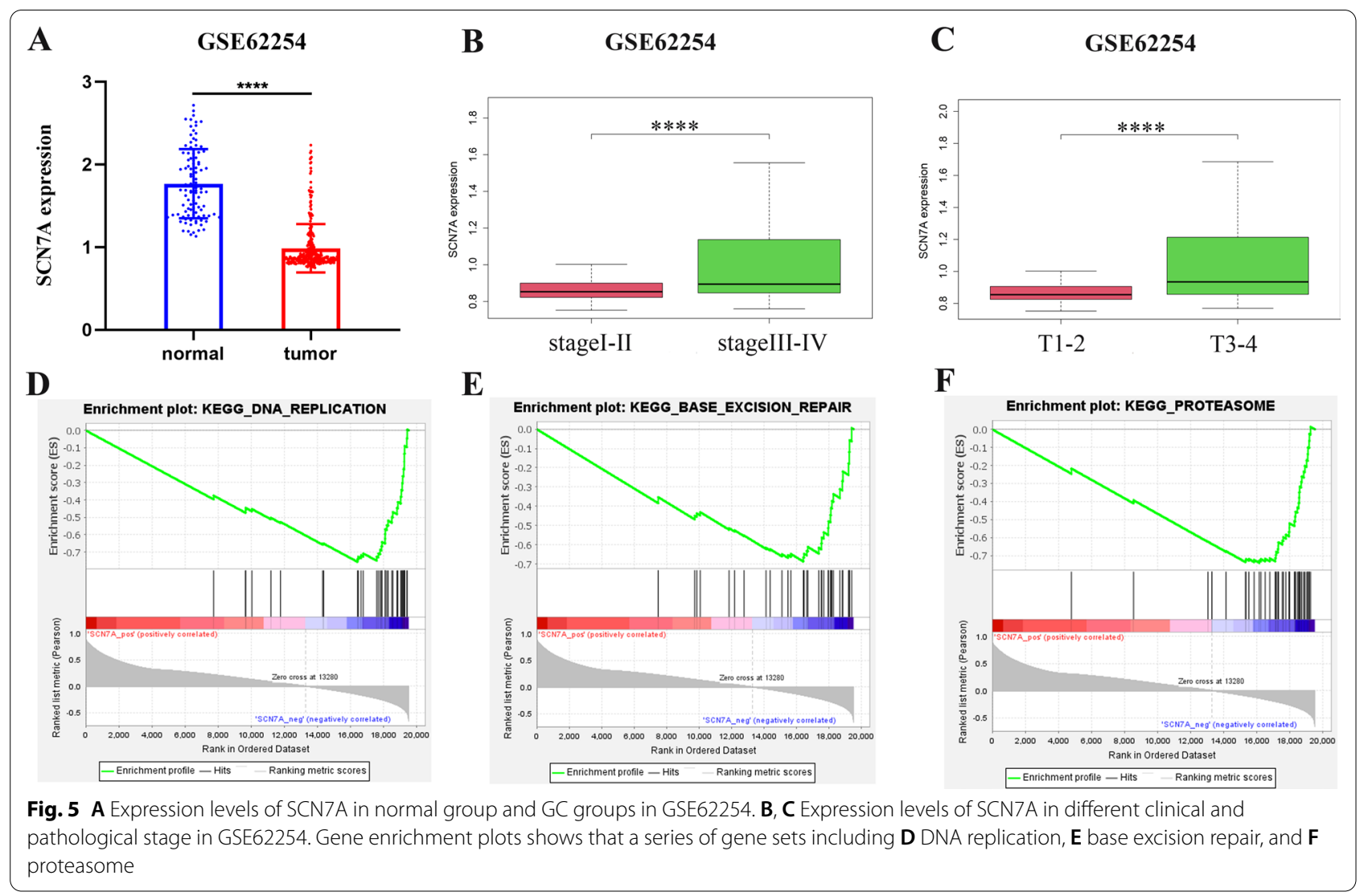

Table 1 Characteristics of patients in GSE62254

\begin{tabular}{|c|c|c|c|c|}
\hline \multirow[t]{2}{*}{ Clinical characteristics } & \multicolumn{2}{|c|}{ SCN7A } & \multirow[t]{2}{*}{ Chi-square } & \multirow[t]{2}{*}{$p$-value } \\
\hline & Low & High & & \\
\hline \multicolumn{5}{|l|}{ Gender } \\
\hline Female & 38 & 63 & 9.329 & $0.002^{* *}$ \\
\hline Male & 112 & 87 & & \\
\hline \multicolumn{5}{|l|}{ Age } \\
\hline$\leq 60$ & 39 & 78 & 21.311 & $<0.001^{* * *}$ \\
\hline$>60$ & 111 & 72 & & \\
\hline \multicolumn{5}{|l|}{ TNM-T } \\
\hline $\mathrm{T} 1-2$ & 113 & 73 & 22.888 & $<0.001^{* * *}$ \\
\hline T3-4 & 36 & 76 & & \\
\hline Tx & 1 & 1 & & \\
\hline \multicolumn{5}{|l|}{ TNM-N } \\
\hline No & 27 & 11 & 7.714 & $0.005^{* *}$ \\
\hline N1-3 & 123 & 139 & & \\
\hline \multicolumn{5}{|l|}{ TNM-M } \\
\hline Mo & 140 & 133 & 1.994 & 0.158 \\
\hline M1 & 10 & 17 & & \\
\hline \multicolumn{5}{|l|}{ Stage } \\
\hline Stage I-II & 79 & 47 & 14.080 & $<0.001^{* * *}$ \\
\hline Stage III-IV & 70 & 102 & & \\
\hline NA & 1 & 1 & & \\
\hline
\end{tabular}

${ }^{* *} p<0.01 ;{ }^{* * *} p<0.001$ tumor development [31]. Therefore, we speculate that low expression of SCN7A improve the prognosis of patient survival by enhancing the response to immunotherapy.

Although much attention has been paid to the role of ion channels in excitable cells, regulation of cell membrane repolarization by ion flow and maintenance of resting potential are also present at the cell membrane of lymphocytes and other immune cells, and are important for maintaining normal electrophysiological function and immune activity [32]. We observed that SCN7A expression was associated with increased memory $\mathrm{CD} 4 \mathrm{~T}$ cell and monocyte infiltration. Activation of CD4+lymphocytes with a memory phenotype enables immune cells to mount a memory response to tumor antigens, thereby preventing tumor recurrence in patients with different cancers, which is predictive of longer survival [33]. As an important part of the innate immune system, mononuclear phagocyte system plays many functions such as immune defense, immune homeostasis, immune surveillance, antigen presentation, and immune regulation in body fluids and tissues [34]. But when tumorigenesis occurs in humans, as CD47 is expressed on the surface of cancer cells, macrophages not only fail to recognize and engulf it but promote it to complete metastasis in the super-early stage [35]. Studies in colon cancer mouse model have found that macrophages 


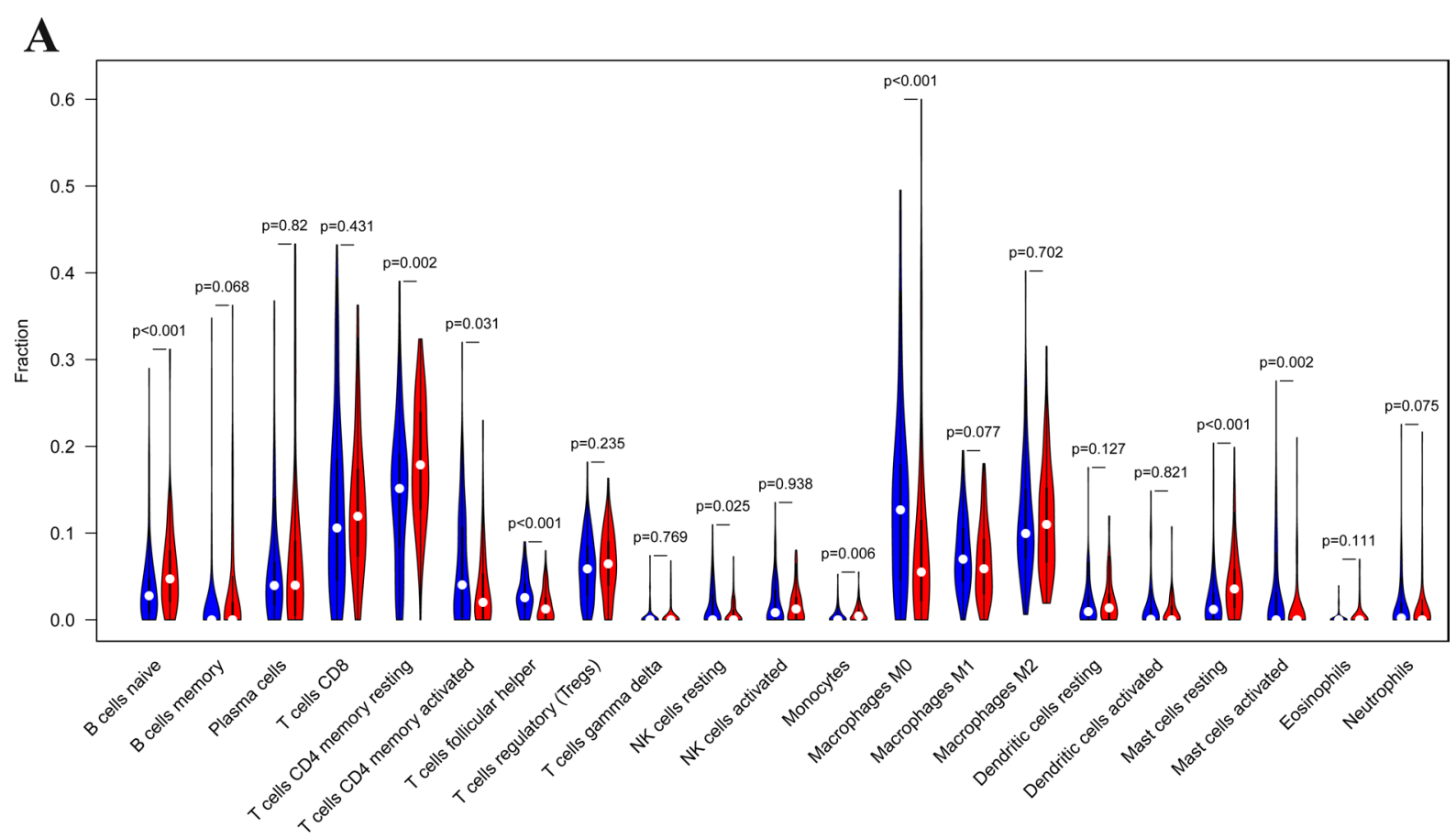

B

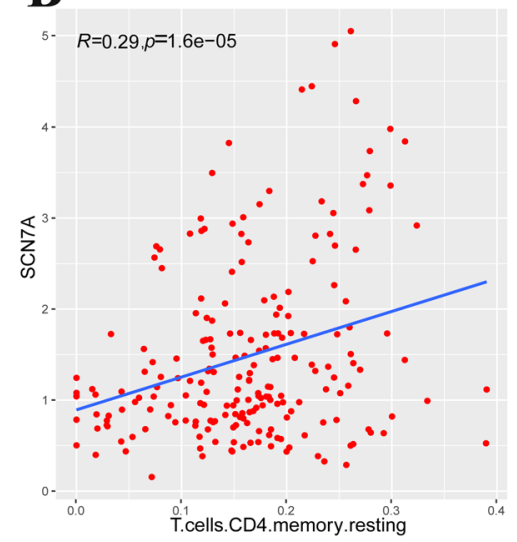

E

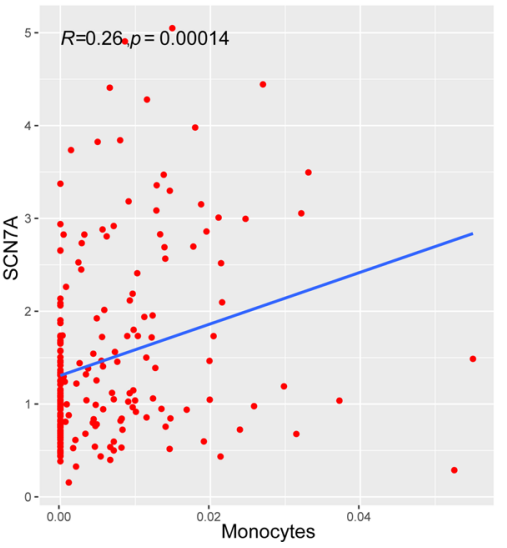

C

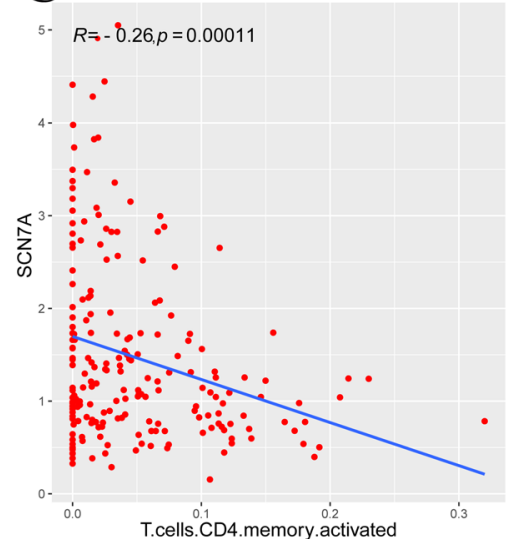

F

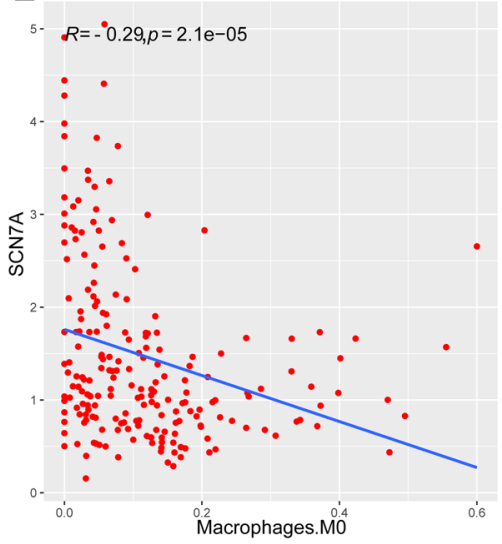

D
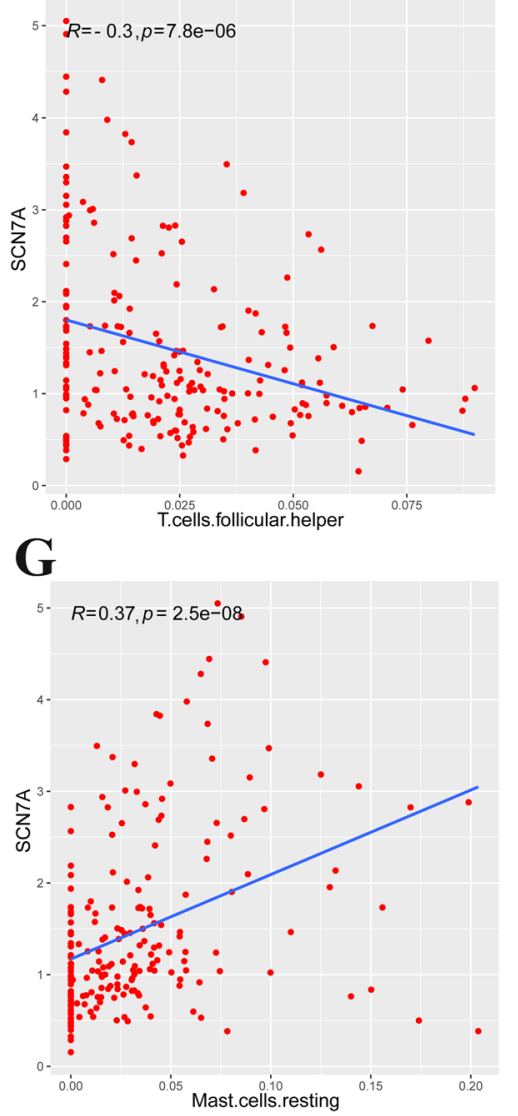

Fig. 6 A The fractions of 21 immune cells between SCN7A low (blue) and high (red) samples. B-G The correlation between SCN7A expression levels and TIICS 
can phagocytose anti-PD-1 antibodies injected into mice, implying that macrophages influence the efficacy of immunochemotherapy drugs [36]. Therefore, we reasoned that expression level of SCN7A cause changes in gastric cancer infiltrating immune cells to promote antitumor immune efficacy.

In conclusion, our study demonstrated that SCN7A mutations in gastric cancer may be associated with higher TMB and improved patient outcomes. In addition, the altered expression of SCN7A affected the signaling pathways of the immune system and affected the antitumor immune response. These findings reveal the possibility that SCN7A is a novel biomarker and able to predict immune responses.

\section{Supplementary Information}

The online version contains supplementary material available at https://doi. org/10.1186/s12876-022-02112-4.

Additional file 1: Figure S2. The process of soft threshold selection through network topology analysis.

Additional file 2: Figure S1. The level of TMB in differnt types of tumors.

\section{Acknowledgements}

Not applicable.

\section{Authors' contributions}

WL, KZ and QZ designed the study. WL, KZ, and ML collected the data. QH, WW and CG analyzed the data. WL, KZ and ML wrote the paper. LL and QZ reviewed the paper. All authors approved the final manuscript.

\section{Funding}

This study was supported by the National Science Foundation of China (No: 81870390; No: 81800494).

\section{Availability of data and materials}

The data sets used and/or analysed during the current study are available from the corresponding author on reasonable request. The public data source: TCGA database (https://portal.gdc.cancer.gov/), GEO database (https://www. ncbi.nlm.nih.gov/geo/) and GEPIA2 database (http://gepia2.cancer-pku.cn).

\section{Declarations}

Ethics and consent to participate

Not applicable.

\section{Consent for publication}

Not applicable.

\section{Competing interests}

The authors declare that there are no conflicts of interest.

\section{Author details}

'Department of Gastroenterology, Zhongnan Hospital of Wuhan University, Wuhan, China. ${ }^{2}$ Hubei Clinical Center and Key Lab of Intestinal and Colorectal Diseases, Wuhan, China.

Received: 17 June 2021 Accepted: 21 January 2022

Published online: 05 February 2022

\section{References}

1. Bray F, Ferlay J, Soerjomataram I, Siegel RL, Torre LA, Jemal A. Global cancer statistics 2018: GLOBOCAN estimates of incidence and mortality worldwide for 36 cancers in 185 countries. CA Cancer J Clin. 2018:68(6):394-424

2. Song M, Rabkin C, Camargo M. Gastric cancer: an evolving disease. Curr Treat Options Gastroenterol. 2018;16(4):561-9.

3. Hudler P. Challenges of deciphering gastric cancer heterogeneity. World J Gastroenterol. 2015:21(37):10510-27.

4. Marrelli D, Polom K, Pascale V, Vindigni C, Piagnerelli R, De Franco L, Ferrara F, Roviello G, Garosi L, Petrioli R, et al. Strong prognostic value of microsatellite instability in intestinal type non-cardia gastric cancer. Ann Surg Oncol. 2016;23(3):943-50.

5. Donato E, Fernández-Zarzoso M, De La Rubia J. Immunotherapy for the treatment of Hodgkin lymphoma. Expert Rev Hematol. 2017;10(5):417-23.

6. Brahmer J, Tykodi S, Chow L, Hwu W, Topalian S, Hwu P, Drake C, Camacho L, Kauh J, Odunsi K, et al. Safety and activity of anti-PD-L1 antibody in patients with advanced cancer. N Engl J Med. 2012;366(26):2455-65.

7. Hodi F, O'Day S, McDermott D, Weber R, Sosman J, Haanen J, Gonzalez R, Robert C, Schadendorf D, Hassel J, et al. Improved survival with ipilimumab in patients with metastatic melanoma. N Engl J Med. 2010;363(8):711-23.

8. Ritterhouse LL. Tumor mutational burden. Cancer Cytopathol. 2019;127(12):735-6

9. Dyck L, Mills K. Immune checkpoints and their inhibition in cancer and infectious diseases. Eur J Immunol. 2017:47(5):765-79.

10. Chan T, Yarchoan M, Jaffee E, Swanton C, Quezada S, Stenzinger A, Peters $\mathrm{S}$. Development of tumor mutation burden as an immunotherapy biomarker: utility for the oncology clinic. Ann Oncol Off J Eur Soc Med Oncol. 2019:30(1):44-56.

11. Samstein R, Lee C, Shoushtari A, Hellmann M, Shen R, Janjigian Y, Barron D, Zehir A, Jordan E, Omuro A, et al. Tumor mutational load predicts survival after immunotherapy across multiple cancer types. Nat Genet. 2019:51(2):202-6.

12. Braun D, Burke K, Van Allen E. Genomic approaches to understanding response and resistance to immunotherapy. Clin Cancer Res Off J Am Assoc Cancer Res. 2016;22(23):5642-50.

13. High TMB predicts immunotherapy benefit. Cancer Discov. 2018;8(6):668.

14. Moehler MH, Cho JY, Kim YH, Kim JW, Di Bartolomeo M, Ajani JA, Yamaguchi K, Balogh A, Kong-Sanchez MT, Bang YJ. A randomized, open-label, two-arm phase II trial comparing the efficacy of sequential ipilimumab (ipi) versus best supportive care (BSC) following first-line (1L) chemotherapy in patients with unresectable, locally advanced/metastatic (A/M) gastric or gastro-esophageal junction (G/GEJ) cancer. J Clin Oncol. 2016:34:4011.

15. Kang YK, Satoh T, Ryu MH, Chao Y, Kato K, Chung HC, Chen JS, Muro K, Kang WK, Yoshikawa T, et al. Nivolumab (ONO-4538/BMS-936558) as salvage treatment after second or later-line chemotherapy for advanced gastric or gastro-esophageal junction cancer (AGC): a double-blinded, randomized, phase III trial. J Clin Oncol. 2017;35(4):2.

16. Langfelder P, Horvath S. WGCNA: an R package for weighted correlation network analysis. BMC Bioinform. 2008;9:559.

17. Nangraj A, Selvaraj G, Kaliamurthi S, Kaushik A, Cho W, Wei D. Integrated PPI- and WGCNA-retrieval of hub gene signatures shared between Barrett's Esophagus and Esophageal Adenocarcinoma. Front Pharmacol. 2020:11:881.

18. Horvath S, Dong J. Geometric interpretation of gene coexpression network analysis. PLoS Comput Biol. 2008;4(8):e1000117.

19. Yip A, Horvath S. Gene network interconnectedness and the generalized topological overlap measure. BMC Bioinform. 2007:8:22.

20. Diboun I, Wernisch L, Orengo C, Koltzenburg M. Microarray analysis after RNA amplification can detect pronounced differences in gene expression using limma. BMC Genomics. 2006;7:252.

21. Yu G, Wang L, Han Y, He Q. clusterProfiler: an R package for comparing biological themes among gene clusters. Omics J Integr Biol. 2012;16(5):284-7.

22. Newman $A M$, Liu CL, Green MR, Gentles AJ, Feng W, Xu Y, Hoang CD, Diehn M, Alizadeh AA. Robust enumeration of cell subsets from tissue expression profiles. Nat Methods. 2015;12(5):453-7. 
23. Widmark J, Sundström G, Ocampo Daza D, Larhammar D. Differential evolution of voltage-gated sodium channels in tetrapods and teleost fishes. Mol Biol Evol. 2011;28(1):859-71.

24. Ruan Y, Liu N, Priori S. Sodium channel mutations and arrhythmias. Nat Rev Cardiol. 2009:6(5):337-48.

25. Catterall W, Goldin A, Waxman S. International Union of Pharmacology. XLVII. Nomenclature and structure-function relationships of voltagegated sodium channels. Pharmacol Rev. 2005;57(4):397-409.

26. Yu F, Catterall W. The VGL-chanome: a protein superfamily specialized for electrical signaling and ionic homeostasis. Sci STKE Signal Transduct Knowl Environ. 2004;2004(253):re15.

27. Xia J, Wang H, Li S, Wu Q, Sun L, Huang H, Zeng M. Ion channels or aquaporins as novel molecular targets in gastric cancer. Mol Cancer. 2017;16(1):54

28. Xu W, Hong S, Zhong A, Xie P, Jia S, Xie Z, Zeitchek M, Niknam-Bienia S, Zhao J, Porterfield D, et al. Sodium channel Nax is a regulator in epithelial sodium homeostasis. Sci Transl Med. 2015;7(312):312ra177.

29. Yuen K, Liu L, Gupta V, Madireddi S, Keerthivasan S, Li C, Rishipathak D, Williams P, Kadel E, Koeppen H, et al. High systemic and tumor-associated IL-8 correlates with reduced clinical benefit of PD-L1 blockade. Nat Med. 2020;26(5):693-8.

30. Schalper K, Carleton M, Zhou M, Chen T, Feng Y, Huang S, Walsh A, Baxi $V$, Pandya $D$, Baradet T, et al. Elevated serum interleukin-8 is associated with enhanced intratumor neutrophils and reduced clinical benefit of immune-checkpoint inhibitors. Nat Med. 2020;26(5):688-92.

31. McGranahan N, Furness A, Rosenthal R, Ramskov S, Lyngaa R, Saini S, Jamal-Hanjani M, Wilson G, Birkbak N, Hiley C, et al. Clonal neoantigens elicit T cell immunoreactivity and sensitivity to immune checkpoint blockade. Science. 2016;351(6280):1463-9.

32. Feske S, Skolnik E, Prakriya M. Ion channels and transporters in lymphocyte function and immunity. Nat Rev Immunol. 2012;12(7):532-47.

33. van Leeuwen E, Sprent J, Surh C. Generation and maintenance of memory CD4(+) T Cells. Curr Opin Immunol. 2009;21(2):167-72.

34. Hume $D$, Irvine K, Pridans $C$. The mononuclear phagocyte system: the relationship between monocytes and macrophages. Trends Immunol. 2019;40(2):98-112.

35. Linde N, Casanova-Acebes M, Sosa M, Mortha A, Rahman A, Farias E, Harper K, Tardio E, Reyes Torres I, Jones J, et al. Macrophages orchestrate breast cancer early dissemination and metastasis. Nat Commun. 2018;9(1):21.

36. Arlauckas S, Garris C, Kohler R, Kitaoka M, Cuccarese M, Yang K, Miller M, Carlson J, Freeman G, Anthony R et al: In vivo imaging reveals a tumorassociated macrophage-mediated resistance pathway in anti-PD-1 therapy. Sci Transl Med 2017, 9(389).

\section{Publisher's Note}

Springer Nature remains neutral with regard to jurisdictional claims in published maps and institutional affiliations.

Ready to submit your research? Choose BMC and benefit from:

- fast, convenient online submission

- thorough peer review by experienced researchers in your field

- rapid publication on acceptance

- support for research data, including large and complex data types

- gold Open Access which fosters wider collaboration and increased citations

- maximum visibility for your research: over $100 \mathrm{M}$ website views per year

At BMC, research is always in progress.

Learn more biomedcentral.com/submissions 